J. Clin. Chem. Clin. Biochem.

Vol. 25, 1987, pp. 393-400

(C) 1987 Walter de Gruyter \& Co. Berlin - New York

\title{
Nebenwirkungen und Verhalten von Noradrenalin und Adrenalin im Plasma beim intravenösen Thyroliberin-Test bei Personen mit normaler und gestörter Schilddrüsenfunktion ${ }^{1}$ )
}

\author{
Von D. Ratge, U. Barthels, H. Wisser
}

Abteilung für Klinische Chemie/Labormedizin, Robert-Bosch-Krankenhaus, Stuttgart, FRG und

\section{J. Chr. Bode}

Abteilung für Gastroenterologie und Endokrinologie am Zentrum für Innere Medizin, Robert-Bosch-Krankenhaus, Stuttgart, FRG

(Eingegangen am 10. September 1986/24. März 1987)

Zusammenfassung: An 14 gesunden Probanden, 23 Patienten mit euthyreoter Struma, 9 Hypothyreoseund 17 Hyperthyreose-Patienten wurde der intravenöse Thyroliberin-Test (TRH-Test) mit $400 \mu \mathrm{g}$ Protirelin (Antepan ${ }^{\circledR}$ ) durchgeführt. Bei 54 der 63 untersuchten Personen (86\%) wurden Nebenwirkungen wie Hitzeund Wärmegefühl, Übelkeit, Harndrang, Schwindel und Kopfschmerzen beobachtet, wobei Häufigkeit und Stärke der Beschwerden eine abnehmende Tendenz in der genannten Reihenfolge zeigten. Die 4 untersuchten Gruppen verhielten sich hinsichtlich der Nebenwirkungen identisch. Kurz nach Injektion von Thyroliberin waren mittlere Anstiege des Blutdruckes von $26 \pm 13 \mathrm{~mm} \mathrm{Hg}$ systolisch und $14 \pm 6 \mathrm{~mm} \mathrm{Hg}$ diastolisch sowie der Herzfrequenz von 7,2 $\pm 6,6 \min ^{-1}$ zu verzeichnen. Im Vergleich zu den Kontrollpersonen waren die basalen Katecholaminkonzentrationen im Plasma bei den Patienten mit euthyreoter Struma und Hyperthyreose erniedrigt und bei den Patienten mit Hypothyreose erhöht. Der Thyroliberin-Test führte zu einer Aktivierung des sympathischen Nervensystems mit Konzentrationsanstiegen von Adrenalin und Noradrenalin, die proportional zu den jeweiligen Basalwerten waren. Zur Auslösung der Blutdruck- und Pulserhöhung waren die Konzentrationsänderungen jedoch zu gering. Maximale Adrenalin- und Noradrenalinkonzentrationen, im Mittel $28 \%$ beziehūngswieișe $21 \%$ höher als die Ausgangswerte, wurden in der 2. bis 4 . Minute nach Thyroliberininjektion beobachtet. Żu diesem Zeitpunkt waren sowohl Erhöhung von Blutdruck und Herzfrequenz als auch Häufigkeit und Intensität der subjektiven Symptome bereits wieder abgeklungen. Wegen des sofortigen Auftretens dieser Nebenwirkungen kann angenommen werden, daß Thyroliberin diese Effekte teilweise zentral oder durch direkte Stimulation am Gefäßsystem auszulösen vermag. Da bei einzelnen Patienten systolisch Blutdruckanstiege bis zu $64 \mathrm{~mm} \mathrm{Hg}$ auftraten, sollte die Indikation zum intravenösen Thyroliberin-Test bei schlecht eingestellten Hypertonikern zurückhaltend gestellt werden.

Side effects and changes in plasma norepinephrine and epinephrine levels after intravenous injection of thyroliberin in subjects with normal and abnormal thyroid function

Summary: 14 normal volunteers, 23 patients with euthyroid goiter, 9 patients with hypothyroidism and 17 patients with hyperthyroidism were injected with $400 \mu \mathrm{g}$ thyroliberin (thyrotropin releasing hormone, TRH). The documented side effects were the same in all the $\mathbf{4}$ groups studied. Subjective symptoms such as flushing, nausea, urinary urgency, dizziness and headache in decreasing sequence were mentioned by $86 \%$ of subjects.

1) Diese Arbeit wurde unterstützt aus Mitteln der Robert-Bosch-Stiftung, Stuttgart.

J. Clin. Chem. Clin. Biochem. / Vol. 25, 1987 / No. 7 
Shortly after thyroliberin injection, a mean increase of $26 \pm 13 \mathrm{~mm} \mathrm{Hg}$ for systolic and $14 \pm 6 \mathrm{~mm} \mathrm{Hg}$ for diastolic blood pressure as well as an increased heart rate by $7.2 \pm 6.6 \mathrm{~min}^{-1}$ was demonstrated. Plasma catecholamines were lowered in patients with euthyroid goiter and hyperthyroidism and raised in patients with hypothyroidism, compared with the controls. Thyroliberin administration was associated with an activation of the sympathoadrenal system. The increments in plasma epinephrine and norepinephrine concentrations were proportional to initial values, but were insufficient to affect blood pressure. The mean increase of $28 \%$ for plasma epinephrine and $21 \%$ for norepinephrine were maximal in the second to the forth minute, where subjective symptoms, blood pressure and heart rate were already decreasing. In view of the rapid onset of the subjective symptoms as well as the chronotropic and the pressor response, thyroliberin may partly exert these effects centrally or directly on the vascular system, independently of catecholamines. Since individual systolic blood pressure increased by as much as $64 \mathrm{~mm} \mathrm{Hg}$, caution is advised in selecting patients with risk factors for testing.

\section{Einführung}

Beim intravenösen Thyroliberin-Test werden von den Patienten häufig leichte und schnell reversible Störungen des Allgemeinbefindens angegeben. Neben Blutdruck- und Herzfrequenzanstieg $(1,2)$ wird über Kopfdruck, 'Schwindel, Hitzegefühl, Herzklopfen, Harndrang und Übelkeit $(3,4)$ berichtet. Das Aus$\mathrm{ma} ß$ dieser Nebenwirkungen ist trotz häufiger Anwendung dieses Testes kaum dokumentiert $(5,6)$ und der Pathomechanismus dieser Effekte ist weithin unbekannt. Die beobachteten Nebenwirkungen sind ähnlich den Symptomen bei Zuständen mit erhöhter Katecholaminsekretion wie bei physischen und psychischen Stresssituationen (7), bei Herzerkrankungen (8) oder auch beim Phäochromocytom (9). Tatsächlich konnten nach intracisternaler Thyroliberin-Applikation bei Ratten eine erhöhte Katecholaminsynthese im Hirn (10) und auch ein verstärktes Ansprechen auf Noradrenalin bei Thyroliberin-Anwesenheit im isolierten Vas-deferens-System (11) nachgewiesen werden. Bei gesunden Personen konnte kurz nach intravenöser Thyroliberin-Gabe ein Anstieg von Noradrenalin im Plasma beobachtet werden (12).

In dieser Arbeit soll die Häufigkeit subjektiv empfundener Nebenwirkungen, Änderungen des systolischen und diastolischen Blutdrucks, der Herzfrequenz und der Katecholamine im Plasma doku- mèntiert und geprüft werden, ob ein Züsammenhang zwischen den Nebenwirkungen beim ThyroliberinTest und dem Anstieg der Katecholaminkonzentration im Plasma besteht. Da Patienten mit Hypothyreose erhöhte und solche mit Hyperthyreose erniedrigte Katecholaminkonzentrationen aưfweisen (13), wurden mögliche Unterschiede in der Beschwerdesymptomatik und dem Katecholaminverbalten bei Patienten mit euthyreoter Struma, bei Hypo- und Hyperthyreose im Vergleich zu einer Kontrollgrippe untersucht.

\section{Patienten und Methodik}

An insgesamt 63 Personen wurde der intravenöse ThyroliberinTest mit 400 $\mu \mathrm{g}$ Thyroliberin (Protirelin, Antepan ${ }^{\circledR}$ ) jeweils zwischen 11 und 13 Uhr durchgeführt. Die 49 Patienten wurden teils aus der Schilddrüsenambulanz, teils aus dem stationären Bereich des Krankenhauses ausgewählt. Die Einordnung in die verschiedenen Gruppen erfolgte aufgrund der klinischen Untersuchung sowie der Ergebnisse der Schilddrüsenhormonbestimmung (Tab. 1). Es wurden nur solche Patienten ausgewählt, die keine weiteren wesentlichen Erkrankungen aufwiesen und mit Medikamenten nicht oder weitgehend nur wegen der Schilddrüsenerkrankung behandelt wurden. Vierzehn Proban= den mit ähnlicher Alters- und Geschlechtsverteilung wie die Patienten bildeten die Kontrollgruppe. Diese Personen nahmen keine Medikamente ein und zeigten bei der vorausgegangenen ärztlichen Untersuchung einen guten Gesundheitszustand. Als zusätzliche Vergleichsgruppe wurden zur Aufdeckung zufälligèr bzw. durch die Injektion verursachter Hormon-, Blutdruck-

Tab. 1. Anzahl, Alter, basale Schilddrüsenhormonkonzentrationen sowie Anstieg des Thyrotropins 30 Minuten naç intravenöser Gabe von $400 \mu \mathrm{g}$ Thyroliberin.

\begin{tabular}{|c|c|c|c|c|c|c|}
\hline Untersuchungsgruppe & $\mathrm{N}$ & $\begin{array}{l}\text { Alter } \\
\text { (Jahre) }\end{array}$ & $\begin{array}{l}\text { Trijod- } \\
\text { thyronin } \\
(\mathrm{nmol} / \mathrm{l})\end{array}$ & $\begin{array}{l}\text { Thyroxin } \\
\text { (nmol/l) }\end{array}$ & $\begin{array}{l}\text { Basales } \\
\text { Thyrotropin } \\
(\mathrm{mU} / \mathrm{l})\end{array}$ & $\begin{array}{l}\Delta \text { Thyrotro- } \\
\text { pin } \\
(\mathrm{mU} / \mathrm{l})\end{array}$ \\
\hline $\begin{array}{l}\text { Kontrollgruppe } \\
\text { Euthyreote Struma } \\
\text { Hypothyreose } \\
\text { Hyperthyreose }\end{array}$ & $\begin{array}{r}14 \\
23 \\
9 \\
17\end{array}$ & $\begin{array}{l}58 \pm 11 \\
44 \pm 12 \\
63 \pm 18 \\
64 \pm 14\end{array}$ & $\begin{array}{l}2,0 \pm 0,6 \\
2,3 \pm 0,7 \\
1,2 \pm 0,6 \\
6,0 \pm 1,2\end{array}$ & $\begin{array}{r}102 \pm 32 \\
97 \pm 33 \\
44 \pm 19 \\
202 \pm 31\end{array}$ & $\begin{array}{c}1,7 \pm 0,9 \\
1,3 \pm 0,5 \\
31 \pm 20 \\
0,9 \pm 0,3\end{array}$ & $\begin{array}{l}10 \pm 7 \\
7,6 \pm 6,2 \\
>50 \\
<0,1\end{array}$ \\
\hline
\end{tabular}


und Pulsschwankungen 6 weitere gesunde Personen im Alter von 23-39 Jahren ausgewählt (14). Nach Aufklärung über Zweck, Ablauf und Nebenwirkungen gaben alle Personen ihr Einverständnis für diese Studie.

Den fortan liegenden Personen wurde eine Butterfly-Kanüle in eine Kubitalvene gelegt, welche durch Spülen mit physiologischer $\mathrm{NaCl}$-Lösung offengehalten wurde. Nach einer 20 minütigen Ruhepause wurden 2 Blutproben im Abstand von 3 Minuten zur Bestimmung der basalen Schilddrüsenhormonkonzentrationen und der mittleren basalen Katecholaminkonzentration entnommen. Sodann erfolgte die rasche Thyroliberin-Injektion, wobei den untersuchten Personen der Zeitpunkt der Thyroliberin-Infusion nicht mitgeteilt wurde. Weitere Blutentnahmen erfolgten $1,2,4,6,8,10,15,30$ und 45 Minuten nach Thyroliberin-Injektion. Parallel zu den Blutentnahmen erfolgten am anderen Arm Blutdruckmessungen nach RivaRocci und Pulsmesssungen durch Auszählung des Radialispulses über 20 Sekunden. Gleichzeitig wurden die angegebenen Beschwerden dokumentiert. Zu den Zeitpunkten 3 Minuten vor und 30 Minuten nach Thyroliberin-Injektion wurden weitere Blutproben zur Bestimmung der Schilddrüsenhormone entnommen (Tab. 1). Nach identischem Versuchsschema wurden der Vergleichsgruppe anstelle von Thyroliberin $10 \mathrm{ml} 0,15 \mathrm{~mol} / \mathrm{l}$ Natriumchloridlösung zügig injiziert.

Die Blutproben von je $2 \mathrm{ml}$ zur Katecholaminbestimmung wurden in eisgekühlte Gefäße überführt, die $40 \mu$ leiner Lösung aus reduziertem Glutathion $(8,8 \mu \mathrm{mol})$ und Ethylenglykol-bis(2-aminoethylether)-N, $\mathrm{N}^{\prime}$-tetraessigsäure (EGTA, $16 \mu \mathrm{mol}$ ) enthielten. Die Proben wurden innerhalb von 30 Minuten bei $4{ }^{\circ} \mathrm{C}$ zentrifugiert und das Plasma bei $-25^{\circ} \mathrm{C}$ bis zur Analyse gelagert.

Noradrenalin, Adrenalin und Dopamin im Plasma wurden mit einer radioenzymatischen Methode von Peuler \& Johnson (15) modifiziert nach Ratge et al. (16) ermittelt. Diese Methode erlaubt den Nachweis von $0,18,0,08$ und $0,20 \mathrm{nmol} / 1$ Noradrenalin, Adrenalin und Dopamin aus $50 \mu$ l Plasma. Die Interassayvariationskoeffizienten bei 12-maliger Bestimmung von Noradrenalin, Adrenalin und Dopamin aus einer Kontrollplasmaprobe betrugen $6,6 \%, 6,9 \%$ und $9,2 \%$.

Die Bestimmung der Schilddrüsenhormone erfolgte radioimmunologisch mit Reagenzien der Firmen Becton \& Dickinson (Heidelberg) für das Trijodthyronin (Gesamt- $\mathrm{T}_{3}$ ) und Thyroxin (Gesamt- $\mathrm{T}_{4}$ ) und der Firma Henning (Berlin) für das Thyrotropin (TSH).

Die Ergebnisse werden als Mittelwerte \pm Standardabweichung angegeben. Die statistische Auswertung erfolgte mittels t- $\bar{T}$ est für verbundene bzw. unabhängige Stichproben sowie durch Regressionsanalysè. Mittels der Varianzanalyse wurde auf Unterșchiede zwischen den Untersuchungsgruppen geprüft.

\section{Ergebnisse}

\section{Beschwerden unter dem Thyroliberin-Test}

Von den 63 untersuchten Persônen gảben 54 (86\%) Beschwerdèn während des Tests an. In der ersten Minute nach Injektion von Thyroliberin waren die Beschwerden am stärksten, wobei die untersuchten Personen in $57 \%$ ein allgemeines Wärmegefühl, in $43 \%$ Harndrang, in $40 \%$ Übelkeit, in $17 \%$ Kopfschmerżen, in $14 \%$ Schwindel und in $3 \%$ Herzklopfen angaben. Die Verteilung der Beschwerden innerhalb der auf jeweils 100 Personen normierten Untersuchungsgruppen ist in Abbildung 1 angegeben. Für

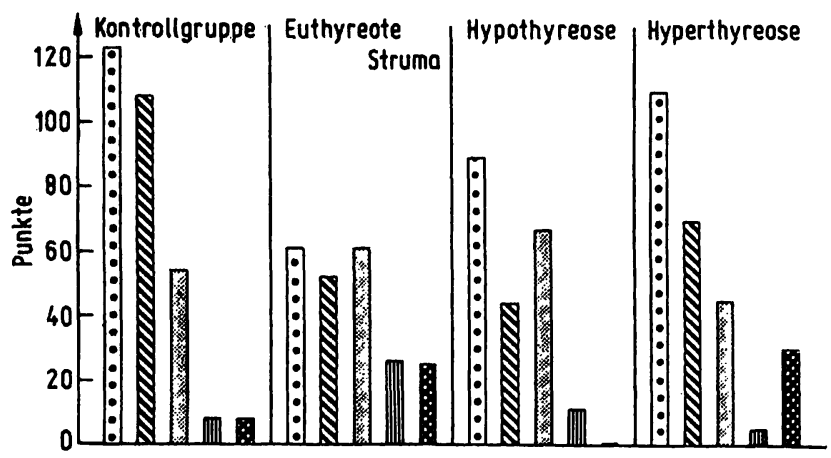

Abb. 1. Beschwerden innerhalb der auf jeweils 100 Personen normierten Untersuchungsgruppen in der 1. Minute nach Thyroliberin-Injektion entsprechend einem Punktesystem, bei dem Häufigkeit und Stärke der Beschwerden berücksichtigt wurden.

曰Wärmegefühl, \$ Harndrang, 畻 Übelkeit, 而 Schwindel, Kopfschmerzen.

jede Gruppe wurde die Zahl der Patienten mit dem jeweiligen Symptom und die subjektiv empfundene Stärke in einem Punktesystem berücksichtigt. Leichte Beschwerden wurden mit dem Faktor 1, mittelstarke Beschwerden mit dem Faktor 2 und starke Beschwerden mit dem Faktor 3 multipliziert. Die Häufigkeit und das Ausmaß der Symptome nahmen schnell wieder $a b$, wie dies exemplarisch für das Symptom „Wärmegefühl" in Abbildung 2 dargestellt ist. In der 10. Minute nach Thyroliberin-Injektion wurden lediglich noch Kopfschmerzen von $3 \%$ und Übelkeit von $2 \%$

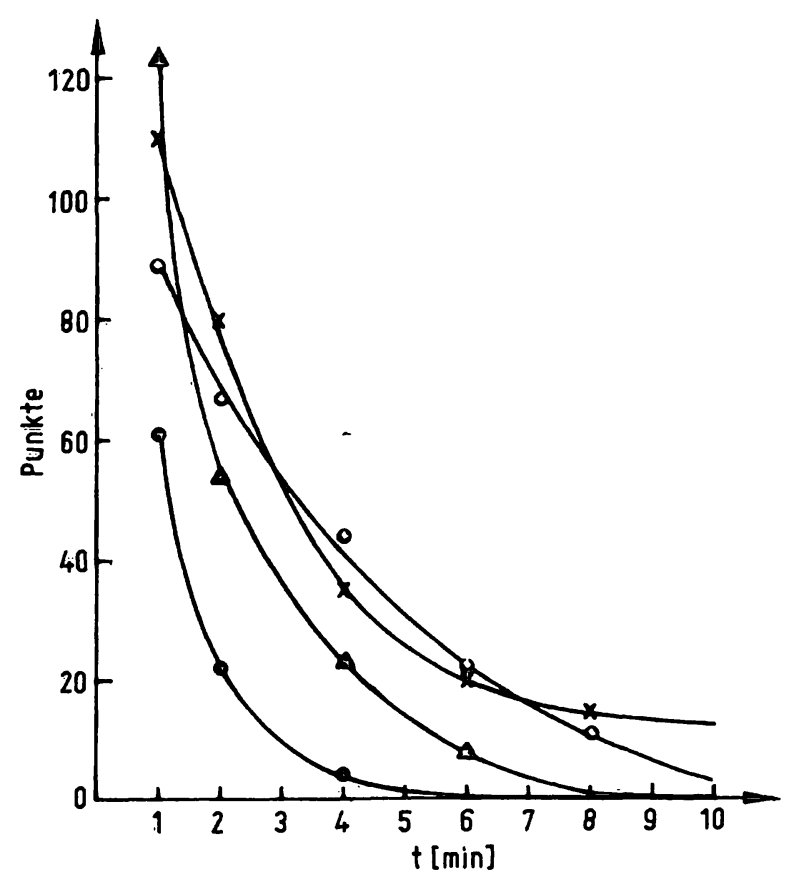

Abb. 2. Zeitlicher Verlauf des Symptoms „Wärmegefühl“" nach Thyroliberin-Injektion bei den auf jeweils 100 Personen normierten Untersuchungsgruppen entsprechend einem Punktesystem.

- euthyreote Struma, $x$ Hyperthyreose, ○ Hypothyreose, $\Delta$ Kontrollgruppe. 
der untersuchten Personen angegeben. Signifikante Unterschiede zwischen den Gruppen hinsichtlich der subjektiven Symptome konnten mit dem $\chi^{2}$-Test zu den Zeitpunkten 1, 2, 4 und 6 Minuten nach Thyroliberin-Injektion nicht festgestellt werden.

Blutdruck- und Herzfrequenzänderungen während des Thyroliberin-Tests

In der ersten Minute nach Thyroliberin-Injektion zeigen die Mittelwerte des systolischen und diastolischen Blutdrucks ebenso wie die Herzfrequenz einen maximalen Anstieg. Signifikante Erhöhungen gegenüber den Ausgangswerten können auch 2 und 4 Minuten nach Thyroliberin-Injektion nachgewiesen werden, wobei die Ruhewerte bereits wieder ab der 6 . bis 8 . Minute erreicht werden (Tab. 2). Bei teils zur Kontrollgruppe signifikant verschiedenen Ruhewerten des Blutdrucks und der Herzfrequenz (Tab. 2) konnte mittels Varianzanalyse im Blutdruck- und Pulsverhalten kein signifikanter Unterschied zwischen den einzelnen Gruppen nachgewiesen werden. Werden die Gruppen zusammengefaßt, so beträgt der maximale Anstieg des systolischen Blutdrucks $26 \pm 13 \mathrm{~mm} \mathrm{Hg}$ (entsprechend einem relativen Anstieg von 19\%), des diastolischen Blutdrucks $14 \pm 6 \mathrm{mmHg}(20 \%)$ und der Herzfrequenz 7,2 $\pm 6,6 \mathrm{~min}^{-1}$ (9\%). Der Bereich der Blutdruck- und Herzfrequenzänderung in der ersten Minute nach Thyroliberin-Injektion war hoch und betrug 5-64 mm $\mathrm{Hg}$ bzw. 0-34 mm $\mathrm{Hg}$ für den systolischen und diastolischen Blutdruckanstieg beziehungsweise $0-16 \mathrm{~min}^{-1}$ für den Herzfrequenzanstieg. Während signifikante Blutdruckanstiege bis zu 4 Minuten nach Thyroliberin-Injektion nachweisbar waren, konnten signifikante Herzfrequenzänderungen schon in der 2. Minute nicht mehr beobachtet werden.

Basale Katecholaminkonzentrationen im Plasma und Veränderungen nach Thyroliberin-Injektion

Gegenüber der Ǩontrollgruppe zeigen Patienten mit euthyreoter Struma und Patienten mit Hyperthyreose signifikant erniedrigte basale Noradrenalin- und Adrenalinkonzentrationen (Tab. 3). Bei Patienten mit Hypothyreose werden erhöhte Noradrenalin- und Adrenalinkonzentrationen ermittelt, die jedoch gegenüber der Kontrollgruppe nicht signifikant verschieden sind. Für alle Untersuchungsgruppen wurden nahezu identische basale Dopaminkonzentrationen in einem Bereich von $217 \pm 112 \mathrm{pmol} / \mathrm{l}$ für die Patienten mit Hypothyreose bis $260 \pm 61 \mathrm{pmol} / 1$ für die Patienten mit Hypothyreose ermittelt.

Thyroliberin verursacht ein rasches Ansteigen der Noradrenalin- und der Adrenalinkonzentration (Tab. 3), ohne die Dopaminkonzentration zu beeinflussen. Für alle Untersuchungsgruppen werden die statistisch

Tab. 2. Verhalten des systolischen, des diastolischen Blutdrucks und der Herzfrequenz in den vier Untersuchungsgruppen beim intravenösen Thyroliberin-Test.

\begin{tabular}{|c|c|c|c|c|c|c|c|}
\hline \multirow{2}{*}{$\begin{array}{l}\text { Kenngröße/ } \\
\text { Untersuchungsgruppe }\end{array}$} & \multicolumn{7}{|c|}{ Zeit nach Thyroliberin-Injektion in Minuten } \\
\hline & 0 & 1 & 2 & 4 & 8 & 15 & 30 \\
\hline \multicolumn{8}{|l|}{$\begin{array}{l}\text { Systolischer } \\
\text { Blutdruck (mm Hg) }\end{array}$} \\
\hline $\begin{array}{l}\text { Kontrollgruppe } \\
\text { euthyreote Struma } \\
\text { Hypothyreose } \\
\text { Hyperthyreose }\end{array}$ & $\begin{array}{l}128 \pm 10 \\
134 \pm 29 \\
134 \pm 31 \\
141 \pm 26\end{array}$ & $\begin{array}{l}163 \pm 17^{* *} \\
158 \pm 33^{* *} \\
155 \pm 37^{* *} \\
164 \pm 32^{* *}\end{array}$ & $\begin{array}{l}160 \pm 17^{* *} \\
150 \pm 33^{*} \\
152 \pm 42^{*} \\
157 \pm 29^{*}\end{array}$ & $\begin{array}{l}149 \pm 16^{*} \\
146 \pm 31^{*} \\
146 \pm 39^{*} \\
150 \pm 28\end{array}$ & $\begin{array}{l}135 \pm 14 \\
136 \pm 28 \\
141 \pm 36 \\
145 \pm 25\end{array}$ & $\begin{array}{l}134 \pm 13 \\
134 \pm 29 \\
140 \pm 37 \\
145 \pm 28\end{array}$ & $\begin{array}{l}133 \pm 14 \\
134 \pm 28 \\
139 \pm 32 \\
145 \pm 28\end{array}$ \\
\hline \multicolumn{8}{|l|}{$\begin{array}{l}\text { Diastolischer } \\
\text { Blutdruck (mm Hg) }\end{array}$} \\
\hline $\begin{array}{l}\text { Kontrollgruppe } \\
\text { euthyreote Struma } \\
\text { Hypothyreose } \\
\text { Hyperthyreose }\end{array}$ & $\begin{array}{l}75 \pm 6 \\
79 \pm 16 \\
76 \pm 11 \\
64 \pm 14^{a}\end{array}$ & $\begin{array}{l}94 \pm 9^{* *} \\
91 \pm 15^{* *} \\
89 \pm 17^{* *} \\
76 \pm 15^{* *}\end{array}$ & $\begin{array}{l}89 \pm 8^{* *} \\
88 \pm 15^{* *} \\
85 \pm 16^{* *} \\
71 \pm 13^{*}\end{array}$ & $\begin{array}{l}83 \pm 6 \\
84 \pm 15^{*} \\
82 \pm 16 \\
67 \pm 12\end{array}$ & $\begin{array}{l}79 \pm 7 \\
81 \pm 15 \\
81 \pm 15 \\
66 \pm 13\end{array}$ & $\begin{array}{l}80 \pm 7 \\
79 \pm 15 \\
79 \pm 15 \\
66 \pm 12\end{array}$ & $\begin{array}{l}79 \pm 6 \\
79 \pm 15 \\
77 \pm 13 \\
66 \pm 12\end{array}$ \\
\hline \multicolumn{8}{|l|}{$\begin{array}{l}\text { Herzfrequenz } \\
\left(\min ^{-1}\right)\end{array}$} \\
\hline $\begin{array}{l}\text { Kontrollgruppe } \\
\text { euthyreote Struma } \\
\text { Hypothyreose } \\
\text { Hyperthyreose }\end{array}$ & $\begin{array}{l}71 \pm 11 \\
72 \pm 15 \\
76 \pm 13 \\
92 \pm 13^{b}\end{array}$ & $\begin{array}{l}82 \pm 9^{* *} \\
81 \pm 17^{* *} \\
83 \pm 17^{*} \\
97 \pm 14^{*}\end{array}$ & $\begin{array}{l}75 \pm 13 \\
75 \pm 16 \\
78 \pm 18 \\
96 \pm 14\end{array}$ & $\begin{array}{l}71 \pm 11 \\
73 \pm 15 \\
74 \pm 16 \\
95 \pm 14\end{array}$ & $\begin{array}{l}68 \pm 11 \\
71 \pm 14 \\
74 \pm 15 \\
93 \pm 14\end{array}$ & $\begin{array}{l}66 \pm 10 \\
68 \pm 12 \\
74 \pm 14 \\
92 \pm 14\end{array}$ & $\begin{array}{l}66 \pm 10 \\
68 \pm 12 \\
73 \pm 15 \\
91 \pm 12\end{array}$ \\
\hline
\end{tabular}

${ }^{*} \mathrm{p}<0,05,{ }^{* *} \mathrm{p}<0,01$ bezogen auf den jeweiligen Ruhewert

${ }^{\mathrm{a}} \mathrm{p}<0,05,{ }^{\mathrm{b}} \mathrm{p}<0,01$ bezogen auf die entsprechenden Ruhewerte der Kontrollgruppe 
Tab. 3. Verhalten der Noradrenalin- und Adrenalinkonzentration im Plasma während des intravenösen Thyroliberin-Testes bei vier Untersuchungsgruppen.

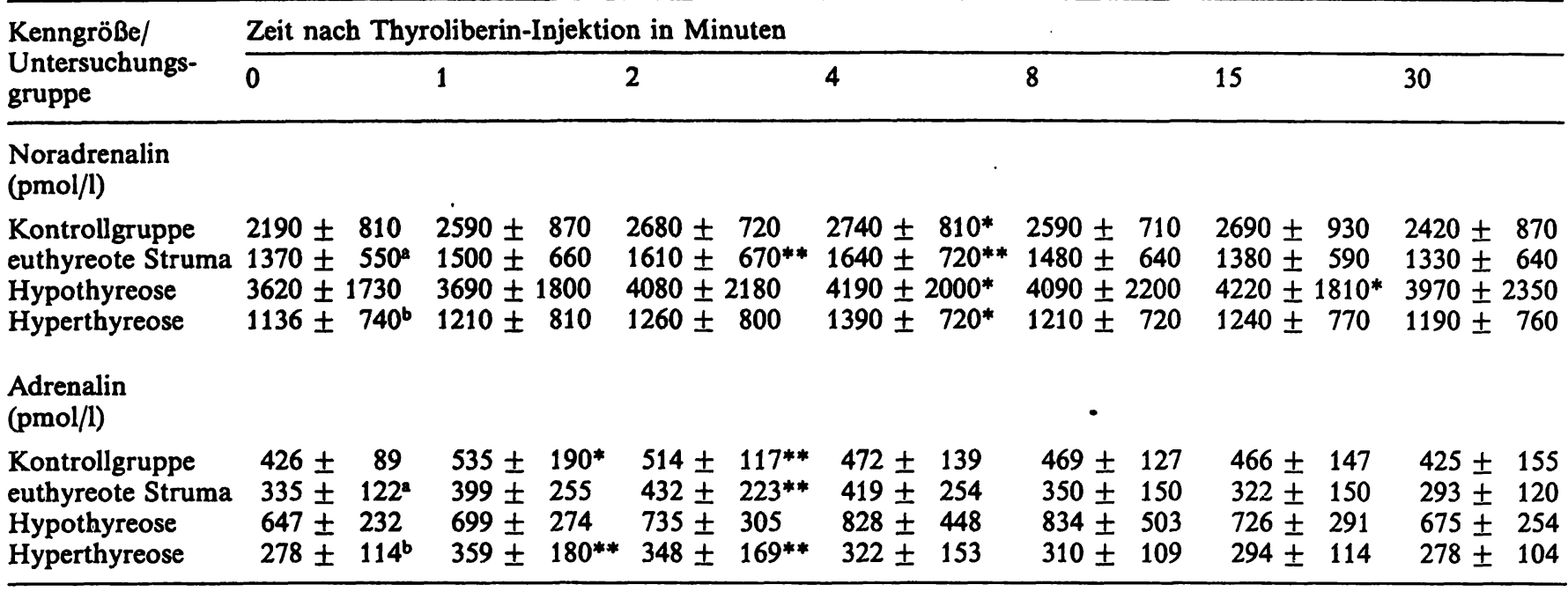

$* \mathrm{p}<0,05,{ }^{* *} \mathrm{p}<0,01$ bezogen auf den jeweiligen Ruhewert

a $\mathrm{p}<0,05,{ }^{\mathrm{b}} \mathrm{p}<0,01$ bezogen auf die entsprechenden Ruhewerte der Kontrollgruppe

gesicherten Maxima für das Noradrenalin in der 4. Minute, für das Adrenalin mit Ausnahme der Hypothyreosegruppe in der 1 . bis 2 . Minute nach Thyroliberin-Injektion erreicht. Annähernd Ausgangswerte werden wieder ab der 10. bis 15 . Minute gemessen. Der absolute Konzentrationsanstieg des Noradrenalins und Adrenalins verhält sich annähernd proportional den jeweiligen Basalkonzentrationen. Das Ausmaß der Konzentrationsveränderungen nach Thyroliberin-Injektion ist für das Adrenalin höher als für das Noradrenalin. Im einzelnen betragen die maximalen Anștiege bezogen auf die jeweiligen Basalwerte für das Noradrenalin und Adrenalin 24 und $26 \%$ für die Kontrollgruppe, 20 und $29 \%$ für die Patienten mit euthyreoter Struma, 16 und $28 \%$ bzw. 22 und $29 \%$ für die Patienten mit Hypo- und Hyperthyreose. Bei den 6 Probanden der Vergleichsgruppe hingegen wurden in den ersten 10 Minuten nach $\mathrm{NaCl}$-Infusion maximale Noradrenalin- und Adrenalinanstiege im Mittel von $14 \%$ und $5 \%$ bezogen auf die Ruhewerte ermittelt, wobei die Veränderungen statistisch nịcht signifikant sind (14).

Korrelation zwischen subjektiven Beschwerden und objektiven Veränderungen

Während Blutdruck- und Herzfrequenzmaxima in allen Gruppen eindeutig in der 1. Minute nach Thyroliberin-Injektion auftreten, sind die Maxima der Noradrenalinkonzentrạtion um etwa 3 Minuten und die Maxima der Adrenalinkonzentration um 0 bis $1 \mathrm{Mi}$ nute hierzu verzögert nachweisbar. Werden die maximalen systolischen und diastolischen Blutdruck- bzw. Herzfrequenzdifferenzen mit den maximalen Nor- adrenalin- und Adrenalindifferenzen korreliert, so ergibt sich mittels linearer Regressionsanalyse für die einzelnen Gruppen als auch unter Einbeziehung aller untersuchten Personen kein signifikanter Zusammenhang. - Zum Zeitpunkt der höchsten Noradrenalin- und Adrenalinkonzentration wird bereits wieder eine deutliche Abnahme der subjektiven Beschwerden registriert. Von 18 der 63 untersuchten Personen ohne bzw. mit geringen Beschwerden zeigten 9 einen Katecholaminanstieg (Noradrenalin- und/ oder Adrenalin) von $<10 \%$ und 9 einen Anstieg von $>10 \%$. Bei 45 Personen mit deutlichen Beschwerden wurden bei 12 ein Katecholaminanstieg von $<10 \%$ und bei 33 ein Anstieg von $>10 \%$ ermittelt. Wird die Intensität der Beschwerden sodann in Beziehung zum Katecholaminanstieg gesetzt, so kann varianzanalytisch nach dem $M c$-Nemar-Test kein statistisch signifikanter Zusammenhang nachgewiesen werden.

Eine analoge Berechnung zwischen der Intensität der Beschwerden bei den Personen und dem systolischen Blutdruckanstieg in der ersten Minute nach Thyroliberin-Injektion von größer bzw. kleiner $13 \%$ bezogen zum Ruhewert zeigt gleichfalls kein statistisch signifikantes Ergebnis.

\section{Diskussion}

Die vorliegende Untersuchung weist nach, daß kurz nach intravenöser Applikation von $400 \mu \mathrm{g}$ Thyroliberin bei zahlreichen Personen eine Reihe von subjektiven Beschwerden auftritt, deren Intensität innerhalb von 10 Minuten exponentiell abnimmt. Der 
systolische und diastolische Blutdruck als auch die Herzfrequenz zeigen einen ähnlichen Verlauf, wobei die Anstiege im Mittel mäßig ausgeprägt sind, im Einzelfall für herzkranke Individuen aber gefährlich sein können. Hierzu um 1-3 Minuten zeitverzögert konnten signifikante Anstiege von Noradrenalin und Adrenalin im Plasma nachgewiesen werden. Alle beobachteten Veränderungen nach Thyroliberin-Injektion waren von der Schilddrüsenfunktionslage unabhängig.

Art und zeitlicher Verlauf der subjektiven Beschwerden bei den hier untersuchten Patienten stimmen mit den Angaben von Andersen et al. (5) und Hershman (3) gut überein. Die Art der häufigsten Beschwerden wie Wärmegefühl und Übelkeit läßt eine zentrale Auslösung dieser Nebenwirkungen vermuten. Ernsthafte Komplikationen wie Bewußtlosigkeit, Krämpfe und Blutdruckabfall, wie sie von Grußendorf et al. (6) und Dolva et al. (17) häufig bei Patienten mit Hypophysenerkrankungen beschrieben wurden, konnten wir nicht beobachten.

Die bei Patienten mit Hyperthyreose erniedrigten und bei Hypothyreose erhöhten basalen Katecholaminkonzentrationen im Vergleich zu gesunden Personen stimmen mit Literaturangaben überein und dienen vermutlich der Kompensation der durch Schilddrüsenhormone veränderten Sensibilität gegenüber den Katecholaminen $(13,18)$. Angaben zu Katecholaminkonzentrationen im Plasma bei Patienten mit euthyreoter Struma sind uns nicht bekannt. Die Ursache der im Vergleich zur Kontrollgruppe signifikant verminderten Noradrenalin- und Adrenalinkonzentration kann nicht mit dem niedrigeren Alter der Patienten mit euthyreoter Struma und der Altersabhängigkeit der Katecholaminkonzentration begründet werden (8). Möglicherweise sind die Unterschiede nur in dieser ausgewählten Stichprobe signifikant. da in einer größeren Kontrollgruppe von uns um $25 \%$ tiefere Noradrenalinkonzentrationen gefunden wurden (16).

Die psychogenen Einflüsse auf die Katecholaminkonzentration im Plasma als Folge der Versuchsanordnung, ermittelt durch Infusion von physiologischer $\mathrm{NaCl}-\mathrm{L} o ̈ s u n g$, sind insbesondere für das Adrenalin weit geringer als die Effekte nach Thyroliberin-Injektion. Bei intravenöser Injektion von $500 \mu \mathrm{g}$ Thyroliberin konnte Morley et al. (4) einen signifikanten Anstieg des Noradrenalins im Plasma bei Probanden nachweisen, während wir zusätzlich einen signifikanten Anstieg des Adrenalins bei 3 von 4 untersuchten Gruppen fanden. Die Konzentrationsanstiege sind bei unterschiedlichster Stimulation des sympathicoadrenalen Systems (19) einschließlich des von uns nach Thyroliberin-Injektion ermittelten Anstiegs proportional den basalen Katecholaminkonzentrationen.

Das Ausmaß des ermittelten maximalen Blutdruckanstiegs stimmt mit den Angaben von Abplanalp (1) und Borowski (2) gut überein und ist etwa doppelt so hoch wie der von Morley et al. (12) mit $9 \mathrm{~mm} \mathrm{Hg}$ angegebene mittlere arterielle Blutdruckanstieg. Der maximale mittlere Anstieg der Pulsfrequenz um 7,2 $\pm 6,6 \mathrm{~min}^{-1}$ war weniger eindrucksvoll und von kürzerer Dauer als der Blutdruckanstieg.

Unsere Untersuchungen geben keine definitive Antwort auf die auslösenden Mechanismen der subjektiven Beschwerden und des Blutdruck- und Herzfrequenzanstieges nach Thyroliberin-Injektion, aber die Ergebnisse sind zusammen mit tierexperimentell gewonnenen Beobachtungen hierzu hilfreich. Dị Auslösung eines Blutdruckanstiegs nach intracisternaler Thyroliberin-Injektion in einer meist geringeren Dosis als dies bei systemischer Applikation notwendig ist (20) bzw. das Ausbleiben des Blutdruckeffekts nach Durchtrennung des Rückenmarks über $\mathrm{Th}_{1}$ (4) sprechen für eine zentrale Wirkung des Thyroliberin. Andererseits bewirkt die intracisternale ThyroliberinApplikation bei der Ratte eine erhöhte Noradrenalinfreisetzung und Syntheserate im Hirn (10) und führt zu erhöhten Katecholaminkonzentrationen im Plasma (21). Wegen der effektiven Blut-HirnSchranke für Katecholamine ist der Übertritt vermehrt gebildeter Katecholamine in den Blutkreislauf ohne Bedeutung (22). Stattdessen macht die Verminderung des Blutdruckeffektes nach intracisternaler Applikation von $10 \mu \mathrm{g}$ Thyroliberin bei mit Phenoxybenzamin vorbehandelten Tieren die Beteiligung des sympathicoadrenalen Systems am Blutdruck- und Plasmakatecholaminanstieg wahrscheinlich (20).

Allerdings ist der ermittelte mittlere Adrenalinanstieg um $28 \%$ ohne jeglichen Einfluß auf den Blutdruck und die Herzfrequenz (23). Das ist auch vom beobachteten Noradrenalinanstieg von im Mittel 21\% anzunehmen, obgleich der im Plasma meßbare Neurotransmitter nur einen Bruchteil des in den synaptischen Spalt sezernierten Noradrenalins darstellt. Wegen der benötigten Zeit für die Diffusion des nicht reabsorbierten Noradrenalins vom synaptischen Spalt in die Zirkulation und die Zeit bis zum Erreichen der Blutentnahmestelle folgen die Anstiege von Noradrenalin und entsprechend weniger ausgeprägt der des Adrenalins dem Blutdruck- und Herzfrequenzanstieg zeitverzögert nach (23). Wegen der zur Erklärung des Blutdruck- und Herzfrequenzanstiegs nicht ausreichenden Höhe des Katecholaminanstiegs im Plasma ist eine zusätzliche direkte Wirkung von Thyroliberin auf blutdruckregulierende Zentren im 
Gehirn anzunehmen. Tatsächlich konnte Thyroliberin mittels immunologischer Methoden in wichtigen kreislaufregulierenden Hirnarealen wie im vorderen Hypothalamus, im Kern des Tractus solitarius und im Kern des Tractus ambiguus nachgewiesen werden (24).

Andererseits erhöht Thyroliberin die Noradrenalinwirkung am isolierten Vas-deferens-System (11) und erhöht möglicherweise die Empfindlichkeit des gesamten Gefäßsystems für geringfügige Konzentrationsänderungen der Katecholamine. Wegen der mäBigen Durchlässigkeit der Blut-Hirnschranke für Thyroliberin (4) und der sofortigen chronotropen und drucksteigernden Effekte nach Thyroliberin-Injektion ist alternativ auch eine direkte katecholaminunabhängige Wirkung von Thyroliberin auf die glatte Muskulatur der Widerstandgefäße des großen Kreislaufs denkbar. Welche der möglichen Mechanismen zur Auslösung der beobachteten Nebenwirkungen wichtig sind, kann momentan nicht angegeben werden. Wegen der mangelnden Korrelation zwischen Katecholamin- und Blutdruckanstieg bzw. der subjektiven Beschwerden scheint die Aktivierung des sympathicoadrenalen Systems hierbei von geringerer Bedeutung zu sein. Eine Abhängigkeit der beobachteten Blutdruck- und Herzfrequenzänderungen von der Thyrotropin-Sekretion ist unwahrscheinlich. Der Thyrotropin-Anstieg beginnt erst 2-5 Minuten nach Thyroliberin-Injektion, zu einem Zeitpunkt also, bei dem die von uns beobachteten Veränderungen bereits wieder abklingen.

Die beobachtbaren Effekte scheinen von der Dosis und der Applikationweise von Thyroliberin abhängig zu sein. Die Blutdruck-, Heržfrequenz- und Katechol- aminanstiege einschließlich der subjektiven Nebenwirkungen sind möglicherweise durch eine hohe lokale Thyroliberin-Konzentration bedingt, wie sie nach rascher Bolusinjektion von $400 \mu \mathrm{g}$ Thyroliberin auftreten. Wegen der schnellen Verteilung und der kurzen Halbwertszeit von Thyroliberin (25) von 4-8 Minuten sind nur flüchtige Effekte beobachtbar. Bereits nach i.v.-Gabe von $200 \mu \mathrm{g}$ Thyroliberin bei gesunden Personen konnten keine Blutdruck- und Herzfrequenzanstiege mehr nachgewiesen werden (26). Nebenwirkungen sind beim oralen und nasalen Thyroliberin-Test nicht beobachtet worden (27). Dies könnte auf die langsamere Resorption von Thyroliberin bei dieser Applikationsweise zurückgeführt werden.

Die subjektiven Beschwerden beim intravenösen Thyroliberin-Test mit $400 \mu \mathrm{g}$ Thyroliberin in dieser Studie bei immerhin $86 \%$ der untersuchten Personen und der Blutdruckanstieg von im Mittel $26 \mathrm{~mm} \mathrm{Hg}$ systolisch und $14 \mathrm{~mm} \mathrm{Hg}$ diastolisch können insgesamt dennoch als unerheblich bezeichnet werden. In Einzelfällen wurden jedoch systolisch Blutdruckanstiege über $50 \mathrm{~mm} \mathrm{Hg}$ registriert. Eine Gefährdung herzinsuffizienter Patienten ist hieraus ableitbar, ebenso wie eine Gefährdung von Patienten mit suboptimal eingestellter Hypertonie durch Auslösung cerebraler Gefäßkomplikationen. Es wird daher empfohlen, bei herzkranken Patienten, bei schlecht eingestellten Hypertonikern und bei Patienten mit Hypophysenerkrankungen $(17,6)$ den nasalen oder oralen Thyroliberin-Test anzuwenden, wenn auf diesen Test trotz Einführung suprasensitiver Thyrotropin-Bestimmungsmethoden und der direkten oder indirekten Messung freier Schilddrüsenhormonkonzentrationen nicht verzichtet werden kann.

\section{Literaturverzeichnis}

1. Abplanalp, H. A. (1976) Arzneim. Forsch. 26, 271-277.

2. Borowski, G. D., Garofano, C. D., Rose, L. J. \& Levy, R. A. (1984) J. Clin. Ëndocrinol. Metab. 58, 197-200.

3. Hershman, J. M. (1974) N. Ënl. J. Med. 290, 886-890.

4. Morley, J. E. (1979) Life Sci. 25, 1539-1550.

5. Anderson, M. S., Bowers, C. Y., Kastin, A. J., Schalch, D. S., Schally, A. V., Snyder, P. J., Utiger, R. D., Wilber, J. F. \& Wise, A. J. (1971) N. Engl. J. Med. 285, 1279-1283.

6. Grußendorf, M., von Blittersdorff, R. \& Hüfner, M. (1982) In: Schilddrüse (Scriba, P. C., Rudorff, K. H. \& Weinheimer, B., eds) pp. 418-429, Georg Thieme Verlag Stuttgart, New York.

7. Dimsdale, J. E. \& Moss, J. (1980) J. Am. Med. Ass. 243, $340-342$.

8. Goldstein, D. S., Lake, C. R., Chernow, B., Ziegler, M. G., Coleman, M. D., Taylor, A. A., Mitchell, J. R., Kopin, I. J. \& Keiser, H. R. (1983) Hypertension 5, 100-104.

9. Manger, W. M. \& Gifford, R. W. Jr. (1977) Pheochromocytoma pp. 88-105, Springer, Berlin-Heidelberg-New York.

10. Agarwal, R. A., Rastogi, R. B. \& Singhal, R. L. C. (1976) Res. Commun. Chem. Pathol. Pharmacol. 15, 743-752.

11. Cuenca, E., Serrano, M. J., Gilbert-Rahola, J. \& Galiana, J. (1975) J. Pharm. Pharmacol. 27, 199-200.

12. Morley, J. E., Tuck, M. L., Mayes, D. M., Rosenblatt, S. \& Hershman, J. (1981) Hormone Res. 14, 18-23.

13. Christensen, N. J. (1979) Acta Med. Scand 45, 9-18.

14. Reimann, I. W., Ratge, D., Wisser, H., Klotz, U. \& Frölich, J. C. (1985) Eur. J. Clin. Pharmacol. 28, 273-277.

15. Peuler, J. D. \& Johnson, G. A. (1977) Life Sci. 21, 625-636.

16. Ratge, D., Baumgardt, G., Knoll, E. \& Wisser, H. (1983) Clin. Chim. Acta 132, 229-243.

17. Dolva, L. Ø., Riddervold, F. \& Thorsen, R. K. (1983) Brit. Med. J. 287, 532-535.

18. Ratge, D., Hansel, S. \& Wisser, H. (1984) Fresenius Z. Anal. Chem. 317, 712-713.

19. Cryer, P. E. (1980) N. Engl. J. Med. 303, 436-444.

20. Horika, A., Carino, M. A. \& Weitzman, R. E. (1979) In: Catecholamines: Basic and Clinical Frontiers, Vol. 2 (Usdin, E., Kopin , I. J. \& Barchas, J., eds.) pp. 897-902, Pergamon, New York. 
21. Brown, M. R. (1981) Life Sci. 28, 1789-1795.

22. Ratge, D., Bauersfeld, W. \& Wisser, H. (1985) J. Neural Transmission 62, 267-284.

23. Kopin, I. J. (1979), In: Catecholamines: Basic and Clinical Frontiers. Vol. 1 (Usdin, E., Kopin, I. J. \& Barches, J., eds.) pp. 897-902, Pergamon, New York.

24. Lux, E. W. Jr., Feuerstein, G. \& Faden, A. J. (1983) Nature $302,822-824$.
25. Griffiths, E. C. \& Kelly, J. A. (1979) Mol. Cell. Endicrinol. $14,3-17$.

26. Hall, R., Amos, J., Garrȳ, R. \& Buston, R. L. (1978) Br. Med. J. 2, 174-178.

27. Mühlen, A., v. z. (1984) Akt. Endokr. u. Stoffw. 5, 52-57.

Prof. Dr. Dr. H. Wisser

Abteilung für Klinische Chemie/Labormedizin

Robert-Bosch-Krankenhaus

Auerbachstraße 110

D=7000 Stuttgart 50 